\title{
Papers Presented at the Annual Meetings of the Knee Society
}

\section{Editorial Comment}

\author{
Mark W. Pagnano MD
}

Published online: 22 October 2009

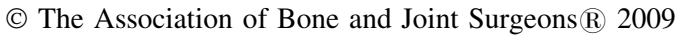

The Papers Presented at the Annual Meetings of the Knee Society Symposium includes selected papers read at the 2008 Members Meeting of the Knee Society held in Denver in October 2008, and the 2009 Open Scientific Meeting of the Knee Society held in Las Vegas in February 2009. The Knee Society education committee, under the able direction of its Program Chair, Tad Vail, MD, assembled an outstanding array of scientific work for presentation at those meetings and it is a privilege to compile for publication many of those papers in this symposium issue. Each paper has gone through an extensive peer review process that is rigorous for the authors but has resulted in a group of manuscripts that are of substantial value for the typical reader. My sincere thanks are extended to the staff at CORR for their timely and valuable hard work in assisting authors through the publication process.

The 2009 Insall Award paper was authored by Dr. Johan Bellemans and colleagues from Belgium, who generated novel data concerning the relationship between patient morphotype and the shape of the distal femur and proximal tibia. Several previous investigators have suggested a relationship between gender and the size and shape of the knee as it relates to fitting the components of a total knee arthroplasty. Other investigators have argued that the differences in shape among men and among women are greater than the differences between those groups. Bellemans and colleagues reviewed data on 1000 patients and proposed a new way to look at this shape difference by focusing on patient morphotype, suggesting morphotype

M. W. Pagnano $(\bowtie)$

Orthopedic Surgery, Mayo Clinic, 200 First Street SW,

Rochester, MN 55905, USA

e-mail: pagnano.mark@mayo.edu may be a more useful way to predict sizing than gender alone.

The 2009 Ranawat Award paper was written by Dr. Sarah Farquhar and colleagues from the University of Delaware and suggests that in the years after unilateral total knee arthroplasty a patient's overall function is strongly correlated to the status of the non-operated contralateral knee. By comparing the function of patients with a unilateral total knee to a group of healthy age-matched controls, these authors were able to discern differences in the function of patients attributable to the operated knee, to the non-operated knee, and those due to age-related declines in function. Such findings are useful as both patients and payors evaluate the relative benefits of costly, but cost-effective, medical interventions such as joint arthroplasty.

Dr. Fabio Catani and colleagues collaborated with the Rizzoli Institute in Bologna, Italy and industry investigators to write the 2009 Coventry Award paper. The authors introduce a novel technique to estimate contact area of the tibiofemoral articulation and of the post-cam articulation of posterior stabilized total knee arthroplasty. By combining in vivo motion data obtained from video fluoroscopy with a finite element model of the total knee components a reproducible technique was devised to calculate the tibiofemoral and post-cam contacts after TKA. In vivo video fluoroscopy has already led to substantial improvements in our understanding of total knee kinematics and further refinements of those techniques may allow the development of more sophisticated predictive models that would allow us to determine if individual patients or specific implant designs might be at particular risk of failure over time.

The remaining papers in this symposium cover a wide range of topics regarding the management of the arthritic knee including autologous chondrocyte implantation, 
unicompartmental and bicompartmental knee arthroplasty, techniques in primary total knee arthroplasty, complications, anesthesia and analgesia techniques, implant design, revision TKA, and outcomes research. The high quality of these papers reaffirms the commitment of the Knee Society to advancing the international orthopaedic community's collective understanding of surgery for the arthritic knee. We hope that readers will find their intellectual curiosity piqued and their clinical practice ultimately improved by the scientific reports in this symposium. 